\title{
Reutilización de la información generada en el sector público español
}

\author{
Rosario Arquero Avilés*, Félix Del Valle Gastaminza*, L. Fernando Ramos Simón*, \\ luliana Botezán*, Concepción Mendo Carmona*, Rodrigo Sánchez Jiménez*, \\ Carlos Tejada Artigas ${ }^{*}$ Silvia Cobo Serrano*, Andrea Sala Jiménez*
}

Resumen: Se presenta el análisis de los resultados del cuestionario dirigido a los organismos del sector público español con la finalidad de caracterizar la información generada por dicho sector susceptible de reutilización, y se analiza el proceso de generación, difusión y comercialización de la misma en el marco de la Ley española 37/2007, de 16 de noviembre, sobre reutilización de la información del sector público (Directiva 2003/98/CE del Parlamento Europeo y del Consejo, de 17 de noviembre de 2003.

Palabras clave: Información publica, reutilización de información, administración pública.

\section{Re-use of public sector information from the perspective of the Spanish public administration}

Abstract: This article analyzes the results of a questionnaire directed to public sector bodies, which sought to characterize public sector information that could be re-used and to study the way in which it is produced, disseminated and commercialized, all in relation to Spain's Law 37/2007 and EU Directive 2003/98/CE, both addressed to the re-use of public sector information.

Keywords: Public information, information re-use, public administration.

\section{Introducción}

La información generada por la administración pública tiene un interés indudable para las empresas, en cuanto a que supone un importante activo para la toma de decisiones en sus ámbitos de actuación, contribuyendo, por tanto, al cre-

* Universidad Complutense de Madrid. Facultad de Ciencias de la Documentación. Departamento de Biblioteconomía y Documentación. MADRID, España. Correo-e: http://multidoc.rediris.es/ppo/ index.php; publidoc@ccdoc.ucm.es.

Recibido: 09-12-10; 2. ${ }^{a}$ versión: 18-01.2011; aceptado: 20-05-2011. 
cimiento económico y a la creación de empleo. Asimismo es un recurso vital para los ciudadanos como elemento de transparencia democrática. La Directiva 2003/98/CE, de 17 de noviembre de 2003, del Parlamento Europeo y del Consejo, relativa a la reutilización de la información del sector público, surge sobre esa base y se adoptó con el objetivo de explotar el potencial de información del sector público y superar las barreras de un mercado europeo fragmentado estableciendo unos criterios homogéneos, asentados en condiciones equitativas, proporcionadas y no discriminatorias para el tratamiento de la información susceptible de ser reutilizada por personas físicas o jurídicas.

La directiva ofrece una primera definición de reutilización de información pública al considerar "... el uso de documentos que obran en poder de organismos del sector público por personas físicas o jurídicas con fines comerciales o no comerciales distintos del propósito inicial que tenían esos documentos en la misión de servicio público para la que se produjeron."

En la exposición de motivos ofrece una serie de razonamientos en los que se explican las causas de índole económica y social que motivan la propuesta. Las líneas argumentales básicas son las de explotar el potencial de la información del sector público y superar las barreras de un mercado europeo fragmentado. En concreto, se destacan los siguientes aspectos:

- La sociedad de la información y del conocimiento afecta a la vida de todos los ciudadanos, al permitirles contar con nuevos medios y productos para acceder y adquirir el conocimiento. La creación y difusión de contenidos digitales constituye por ello un potente motor del crecimiento, la competitividad y el empleo. La reutilización de la información del sector público contribuirá al logro de esos objetivos.

- El sector público recoge, trata y difunde gran cantidad de información:

- Información comercial y financiera.

- Jurídica y administrativa.

- Geográfica, sobre tráfico, turística, etc.

- Para los ciudadanos y las empresas resultará de gran interés disponer de esta información a través de Internet, además, facilitará la comunicación con las administraciones públicas y la participación en el proceso democrático.

- Esta información es esencial para muchos productos de información digital. Se trata de un activo importante en el sector de contenidos digitales, un sector que ya da empleo a cuatro millones de ciudadanos.

- La integración de las economías europeas generará una demanda de productos y servicios de información paneuropea. En la actualidad, la creación de este tipo de productos y servicios choca con unas normas y prácticas diferentes en cada Estado, lo que crea incertidumbre sobre las condiciones de uso a los organismos públicos, a las empresas y a los ciudadanos. 
- Al establecer un conjunto mínimo de normas comunes se crearán las condiciones que harán posible la creación de productos para toda la Unión. Al mismo tiempo, los organismos públicos podrán contar con unos criterios comunes cuando reciban solicitudes de reutilización de la información.

- La Directiva no afectará negativamente el acceso a los documentos por parte de los ciudadanos, ni modificará las actuales normas de acceso a los documentos de los Estados miembros. Asimismo, la directiva pretende favorecer el logro de los objetivos del mercado interior definido en el art. 95 del Tratado.

- Por último, en cuanto a los precios y el problema de tarificación de la información del sector público, la cuestión se debate entre un modelo de bajo coste como el aplicado en Estados Unidos en el que las tarifas no superan los costes marginales de reproducción y difusión y otros modelos de recuperación de costes utilizados en Europa. La propuesta tiene en cuenta que algunos organismos públicos dependen de los ingresos procedentes de la venta de sus recursos para financiar parte de sus actividades, por lo que no impone modificación en esas políticas, pero insta a los organismos públicos a que adopten el enfoque del coste marginal de reproducción y difusión cuando sea posible.

La incorporación de la Directiva al ordenamiento jurídico español tiene lugar a través de la Ley 37/2007, de 16 de noviembre, sobre reutilización de la información del sector público. La Ley considera reutilización el uso de documentos que obran en poder de las Administraciones y organismos del sector público, por personas físicas o jurídicas, con fines comerciales o no comerciales, siempre que dicho uso no constituya una actividad administrativa pública. Excluye del concepto el intercambio de documentos entre Administraciones y organismos del sector público en el ejercicio de las funciones públicas que tengan atribuidas.

Con objeto de revisar la aplicación de la Directiva y resaltar el potencial de reutilización de esta información en la era digital, la Comisión presentó en 2009 una Comunicación en la que ponía en evidencia la existencia de una serie de barreras para el acceso y empleo de la información pública, citando específicamente la existencia de acuerdos exclusivos, prohibidos expresamente por la Directiva y determinaba poner en marcha una investigación sobre el alcance de tales acuerdos en el verano de 2009.

Esta investigación fue encargada a distintos organismos e investigadores de diversos países de la Unión Europea y, en concreto, el estudio de la situación española fue objeto de un contrato entre la Comisión y el Grupo Publidoc, dirigido por el profesor de la Universidad Complutense de Madrid D. L. Fernando Ramos Simón. Como resultado de esa investigación, los análisis realizados en ocho países de la Unión Europea, publicados en el Portal de la Sociedad de la Información de la Comisión, y de los cuales publicamos aquí el análisis de los datos obtenidos desde la óptica de las administraciones públicas relativos a España, han tratado de identificar la permanencia de determinadas barreras al acceso y empleo de los datos públicos, en un doble sentido: 
- Nivel real de conocimiento de la Directiva y grado de concienciación adquirido sobre el potencial de la actividad reutilizadora.

- Posibles prácticas discriminatorias, actuaciones monopolísticas o falta de transparencia y claridad en la apertura de dichos recursos.

El estudio ha sido realizado por el Grupo Publidoc de la Universidad Complutense de Madrid (UCM) y en él se establecen similares recomendaciones a las plasmadas en los restantes estudios de Austria, Bélgica, Dinamarca, Francia, Italia, Polonia y República Checa, centradas en reforzar la divulgación de los beneficios socioeconómicos de la apertura de la información del sector público y potenciar la difusión de las actuaciones derivadas de la Ley 37/2007, de 16 noviembre.

\section{Material y métodos}

Se presenta en el trabajo el análisis de los resultados de la administración de un cuestionario dirigido a los organismos del sector público y que ha tenido como finalidad caracterizar la información del sector público español susceptible de reutilización y analizar el proceso de generación, difusión y comercialización de la misma en el marco de la Ley española 37/2007, de 16 de noviembre, sobre reutilización de la información del sector público (Directiva 2003/98/CE del Parlamento Europeo y del Consejo, de 17 de noviembre de 2003, relativa a la reutilización de la información del sector público).

Hemos de señalar que la realización de este cuestionario, se enmarca en un trabajo de investigación más amplio que tenía como objetivo identificar situaciones que constituyeran acuerdos exclusivos o pudieran, potencialmente, ser considerados como tales en el contexto de la Directiva 2003/98/CE. Los principales resultados de dicho trabajo de investigación se publicaron en el informe "PSI: Identification of Potential Exclusive Agreements - Spain/Final Report", elaborado en 2010 por el Grupo Publidoc. Ahora bien, dado que el formato y las características de publicación de los informes elaborados para la Comisión Europea por los distintos países (cuya relación presentamos en la sección "Estudios sobre Acuerdos exclusivos elaborados para la Comisión Europea", en el apartado "Fuentes y bibliografía"), no contemplaba la publicación de los resultados concretos del cuestionario a organismos de la Administración Pública, nos parece relevante difundir, expresamente en este trabajo, los hallazgos derivados de la realización del cuestionario dirigido a organismos del sector público en España y que constituyen resultados originales de la investigación.

En virtud de lo señalado, hemos de poner de manifiesto que, en el caso español, se ha utilizado un cuestionario semiestructurado de 24 preguntas que se engloban en cuatro bloques. En el primer bloque se abordan las condiciones iniciales para la reutilización de la información del sector público en cada organismo. Las preguntas se centran en indagar las características de la producción y de la política de difusión, distribución y, en su caso, venta, de la información 
potencialmente reutilizable. En el segundo bloque se analizan las características de la información que es generada y distribuida con fines de reutilización (tipologías y clases de información, procedencia de la misma, formatos, profesionales que participan en su generación, solicitud, tasas o precios públicos y público objetivo de dicha información). En el tercer bloque se indaga sobre los acuerdos de explotación y las condiciones de uso de la información reutilizable (existencia de acuerdos de reutilización, existencia de acuerdos exclusivos e información disponible sobre acuerdos exclusivos y requisitos y condiciones de uso de dicha información). Finalmente, se incorpora un cuarto bloque para la inclusión de los datos de clasificación del organismo.

El diseño de la encuesta se ha sustentado:

- En la discusión y análisis por parte del Grupo de Investigación Publidoc para la identificación de las dimensiones del cuestionario y la elección de la tipología de preguntas, formato de respuestas, estructuración en los bloques mencionados, selección del público objetivo de la encuesta y procedimientos de administración de la misma.

- En el análisis de cuestionarios utilizados en estudios previos sobre reutilización de información del sector público y en la adaptación de su orientación y contenido al contexto español.

El grupo de investigación envió el cuestionario a 101 organismos del sector público que representaban los seis grandes sectores del mercado de la información pública en los que se han basado todos los estudios sobre acuerdos exclusivos elaborados para la Comisión Europea y cuya relación se presenta en el apartado "Fuentes y bibliografía". La distribución según estos sectores se ha basado en una descripción del mercado de la información pública en términos de actividad, tanto en la producción y difusión de los activos de información (sector público) como en la reutilización de la misma (sector privado). Para determinar la importancia de cada sector, se analizaron los datos relativos a la disponibilidad de productos y activos de información del sector público (en síntesis, bases de datos, conjuntos de datos y publicaciones oficiales tanto de la Administración General del Estado como de las Comunidades Autónomas). El análisis de disponibilidad de dichos productos y activos de información se sustenta en un estudio detallado sobre el concepto de activos de información pública que se realizó en un trabajo previo (Ramos Simón y otros, 2009).

El cuestionario se envió por e-mail e iba precedido de un protocolo de presentación (con una explicación del mismo) así como una carta de la Comisión Europea en la que se invitaba a participar en el estudio y que mostraba el apoyo de dicha institución a Publidoc, como grupo de investigación encargado de su realización.

El envío del cuestionario se complementó con llamadas telefónicas para solicitar información adicional, localizar a la persona adecuada o para entrevistar directamente al personal y completar el cuestionario. La tabla I muestra un es- 
quema de la tasa de respuesta y el número de cuestionarios enviados por sectores de mercados de información.

\section{TABLA I}

Cuestionarios enviados por sectores del mercado de la información pública $y$ ratios de respuesta

\begin{tabular}{l|c|c|c}
\hline Sectores del mercado de la información & \multicolumn{1}{c|}{$\begin{array}{c}\text { Cuestionarios } \\
\text { enviados }\end{array}$} & $\begin{array}{c}\text { Respuestas } \\
\text { recibidas }\end{array}$ & \% \\
\hline Negocios/Información económica & 38 & 17 & 44,74 \\
\hline Información geográfica & 7 & 4 & 57,14 \\
\hline Información legal & 13 & 6 & 46,15 \\
\hline Información meteorológica & 3 & 3 & 100 \\
\hline Datos sociales & 34 & 13 & 38,24 \\
\hline Información de transportes & 6 & 4 & 66,67 \\
\hline Total & 101 & 47 & $\mathbf{4 6 , 5 3}$ \\
\hline
\end{tabular}

\section{Resultados y discusión}

\subsection{Condiciones iniciales de reutilización de información del sector público}

La mayoría de las instituciones que han respondido afirman ser conscientes de la existencia de un mercado de reutilización de la información que producen (90,48\%), sólo un porcentaje muy pequeño no tiene conciencia de ello (9,52\%).

En relación con la cuestión anterior, sorprende que sólo el 58,54\% disponga de una política de difusión de la información que produce.

La información susceptible de reutilización se difunde preferentemente a través de la Web institucional y, en menor medida, a través de folletos informativos y correos electrónicos, en este orden. En el apartado "Otros medios u otros Websites", destacan las sedes electrónicas de instituciones públicas en general, intranets e incluso el correo ordinario (tabla II).

A pesar de la mayoritaria respuesta afirmativa a la primera cuestión planteada sobre la existencia de un mercado de reutilización de la información que producen los organismos del sector público, la casi totalidad de los encuestados no muestran información relativa a la normativa nacional o comunitaria que regula la reutilización de la información del sector público (una referencia explícita a la Ley 37/2007, de 16 de noviembre, sobre reutilización de la información del sector público), sólo un 10,26\% ofrecen información sin la posibilidad de enlazar con la URL de la norma referida y ninguna institución manifiesta ofrecer el enlace a la ley (tabla III). 
TABLA II

Medios específicos a través de los que se difunde la existencia de información susceptible de reutilización

\begin{tabular}{l|c|c}
\hline & \% & Total \\
\hline E-mail. & 45 & 18 \\
\hline Folletos informativos. & 55 & 22 \\
\hline Internet (a través de su propia página Web). & 87,5 & 35 \\
\hline $\begin{array}{l}\text { A través de la página Web de otra/s entidad/es (indique su denominación } \\
\text { y su URL) o a través de otros medios. }\end{array}$ & 25 & 10 \\
\hline
\end{tabular}

Organismos que han respondido a esta pregunta: 40 .

TABLA III

Existencia de una referencia explícita a la Ley 37/2007, de 16 de noviembre, sobre reutilización de la información del sector público

\begin{tabular}{l|c|c}
\hline & $\mathbf{\%}$ & Total \\
\hline Sí, con URL a la ley. & 0 & 0 \\
\hline Sí, sin URL. & 10,26 & 4 \\
\hline No. & 89,74 & 35 \\
\hline Total & 100 & 39 \\
\hline
\end{tabular}

A la luz de las respuestas recibidas, la mayor parte de los organismos del sector público $(67,5 \%)$ no distribuyen o venden directamente sus productos informativos con fines comerciales.

En relación con las respuestas afirmativas de la pregunta anterior (el 32,5\%), el $76,92 \%$ de los organismos del sector público distribuye o vende directamente sus productos de información (tabla IV). El resto de los organismos han creado una entidad específica. Como tales, se indican los Centros de Publicaciones y se incluyen de forma específica la Oficina de Patentes y Marcas y el Museo Nacional del Prado Difusión S.L.

\subsection{Características de la información generada y distribuida con fines de reutilización}

Los tres sectores de mercado que encabezan la relación de dominios de información generada para ser reutilizada son el de los datos sociales, la información económica y la información legal. Hemos de señalar que el sector de la 
TABLA IV

Vía a través de la que realizan la venta o distribución de productos de información a reutilizadores o empresas con fines comerciales

\begin{tabular}{l|c|c}
\hline & $\%$ & Total \\
\hline $\begin{array}{l}\text { El organismo del sector público vende o distribuye directamente la infor- } \\
\text { mación. }\end{array}$ & 76,92 & 10 \\
\hline Se ha creado una entidad específica para vender nuestra información. & 23,08 & 3 \\
\hline Total & 100 & 13 \\
\hline
\end{tabular}

información económica se señala también, expresamente, como decisivo en el mercado de la información pública en Italia y Francia (la sección "Estudios sobre Acuerdos exclusivos elaborados para la Comisión Europea", en el apartado "Fuentes y bibliografía"). En menor medida, se genera y distribuye en España información, con fines de reutilización (figura 1), sobre transportes, meteorológica e información geográfica (en contraste, según se deriva del análisis de los informes correspondientes, en Italia, Francia y Alemania esta última categoría de información constituye un sector líder en sus mercados de información pública).

Destaca la gran variedad de clases y formatos de presentación de información que ofrece el sector público a sus potenciales reutilizadores (véanse tabla $\mathrm{V}$ y figura 2). Como otras tipologías de información generada se señalaron, en sínte-

\section{FIGURA 1}

Dominios de información generada en los organismos públicos para su reutilización

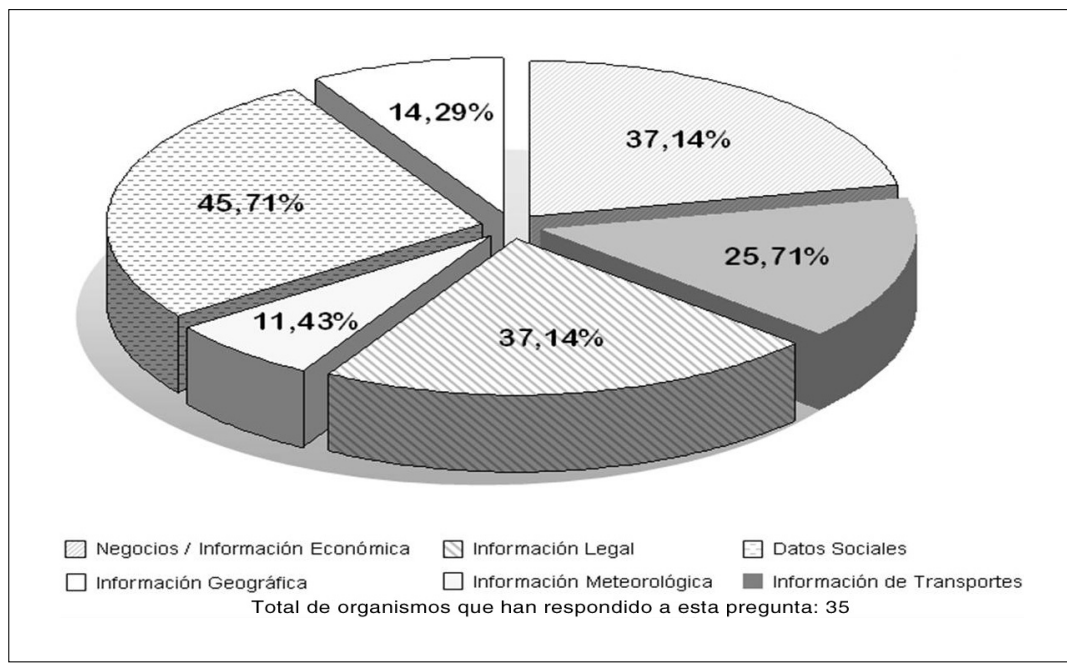


sis, las siguientes: información y material audiovisual, documentación parlamentaria, documentación para la formación, información cultural, normas técnicas y datos de opinión. En lo referente a formatos adicionales de presentación de la información generada para su reutilización se designaron adicionalmente: programas y ficheros informáticos, carteles, artículos científicos y libros.

TABLA V

Clase de información generada para su reutilización

\begin{tabular}{l|c|c}
\hline & $\%$ & Respuestas \\
\hline Datos hidrográficos & 8,11 & 3 \\
\hline Datos históricos & 27,03 & 10 \\
\hline Energía & 13,51 & 5 \\
\hline Estadísticas culturales & 16,22 & 6 \\
\hline Estadísticas de ciencia y tecnología & 24,32 & 9 \\
\hline Estadísticas de consumo & 13,51 & 5 \\
\hline Estadísticas de educación & 5,41 & 2 \\
\hline Estadísticas de empleo & 8,11 & 3 \\
\hline Estadísticas de la Administración Pública & 21,62 & 8 \\
\hline Estadísticas de población & 8,11 & 3 \\
\hline Estadísticas económicas e industriales & 16,22 & 6 \\
\hline Estadísticas sociales & 18,92 & 7 \\
\hline Estado de las carreteras & 2,70 & 1 \\
\hline Estado del tráfico & 5,41 & 2 \\
\hline Fotos aéreas & 16,22 & 6 \\
\hline Información cartográfica & 24,32 & 9 \\
\hline Información catastral & 8,11 & 3 \\
\hline Información de direcciones & 13,51 & 5 \\
\hline Información financiera & 10,81 & 4 \\
\hline Información geológica & 5,41 & 2 \\
\hline Información sanitaria y estadística & 8,11 & 3 \\
\hline Información topográfica & 8,11 & 3 \\
\hline Jurisprudencia & 10,81 & 3 \\
\hline Legislación & 21,62 & 27,03 \\
\hline Licitaciones & 8,11 & \\
\hline Patentes y marcas & & 5 \\
\hline
\end{tabular}


TABLA V (continuación)

\begin{tabular}{l|c|c}
\hline & \% & Respuestas \\
\hline Previsiones climatológicas & 8,11 & 3 \\
\hline Registros de empresas & 10,81 & 4 \\
\hline Sector primario (agricultura, pesca, etc) & 10,81 & 4 \\
\hline Telecomunicaciones & 5,41 & 2 \\
\hline Transporte público & 8,11 & 3 \\
\hline Otras tipologías & 40,54 & 15 \\
\hline
\end{tabular}

Organismos que han respondido a esta pregunta: 37

FIGURA 2

Formatos de presentación de la información generada para su reutilización

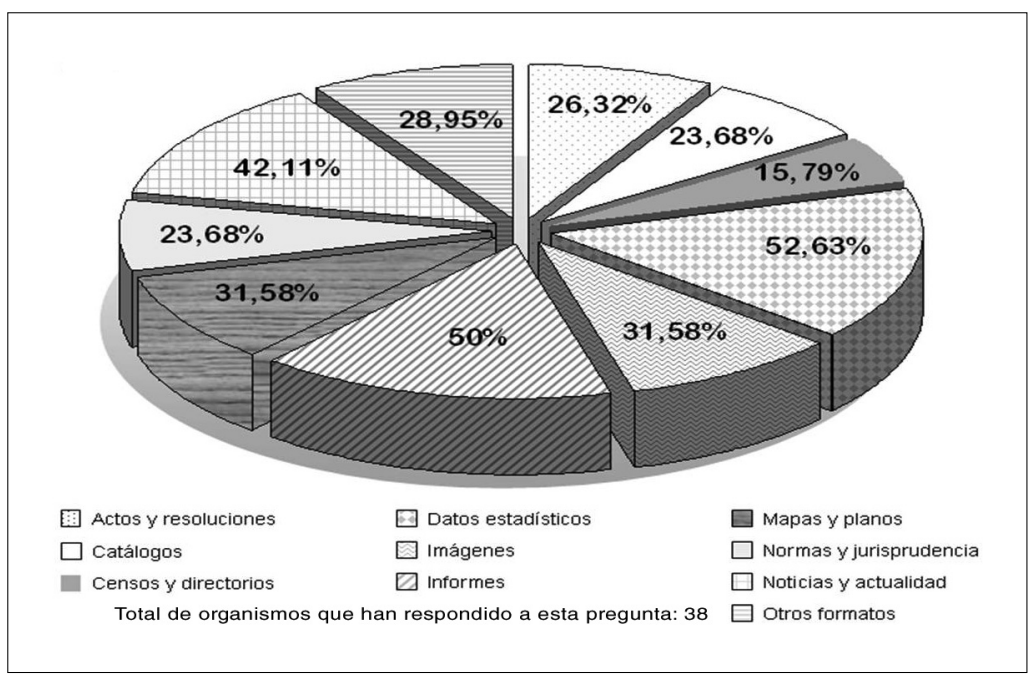

La información que generan, distribuyen y comercializan los organismos del sector público con fines de reutilización procede mayoritariamente de la propia administración pública (figura 3) y, en menor medida, es generada por los ciudadanos, las empresas y otras entidades sin ánimo de lucro.

En el proceso de elaboración de la información reutilizable participa una amplia gama de profesionales procedentes del ámbito de las Ciencias Sociales y Jurídicas, del ámbito Bio-sanitario y de la Ingeniería. Específicamente, los profesionales de la información y documentación se designan como pieza clave en dicho proceso (figura 4). En la categoría de respuesta "Otros" a esta pregunta de res- 


\section{FIGURA 3}

Procedencia de la información generada, distribuida y/o comercializada para su reutilización

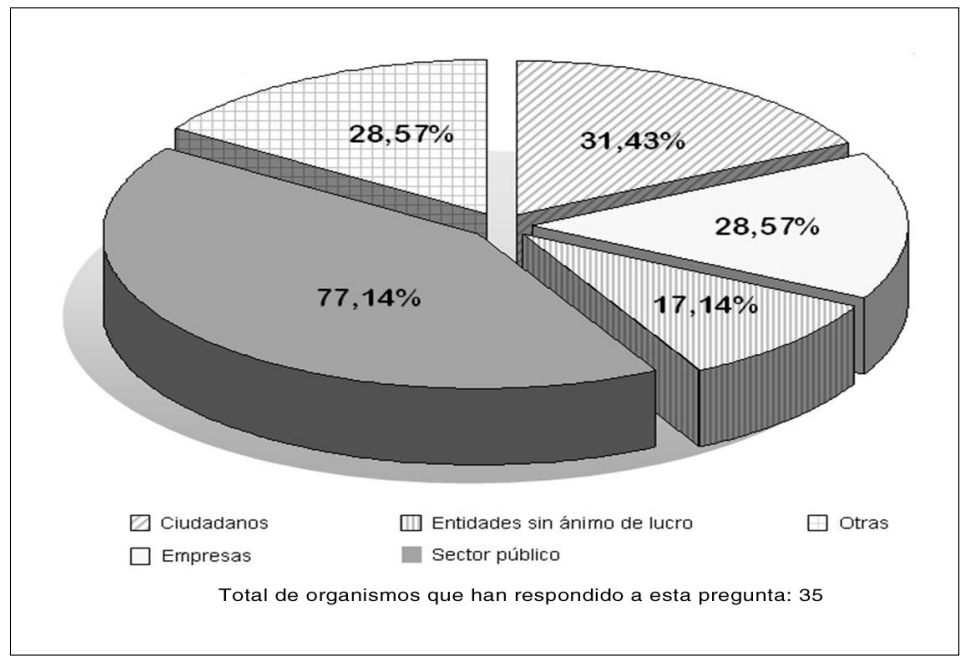

puesta múltiple, se ha hecho referencia explícita a la participación en dicho proceso de los siguientes profesionales: ingenieros, veterinarios, meteorólogos, fotógrafos, geólogos, filólogos, historiadores, biólogos, sociólogos, jueces y magistrados.

\section{FIGURA 4}

Profesionales que participan en el proceso de generación de información reutilizable

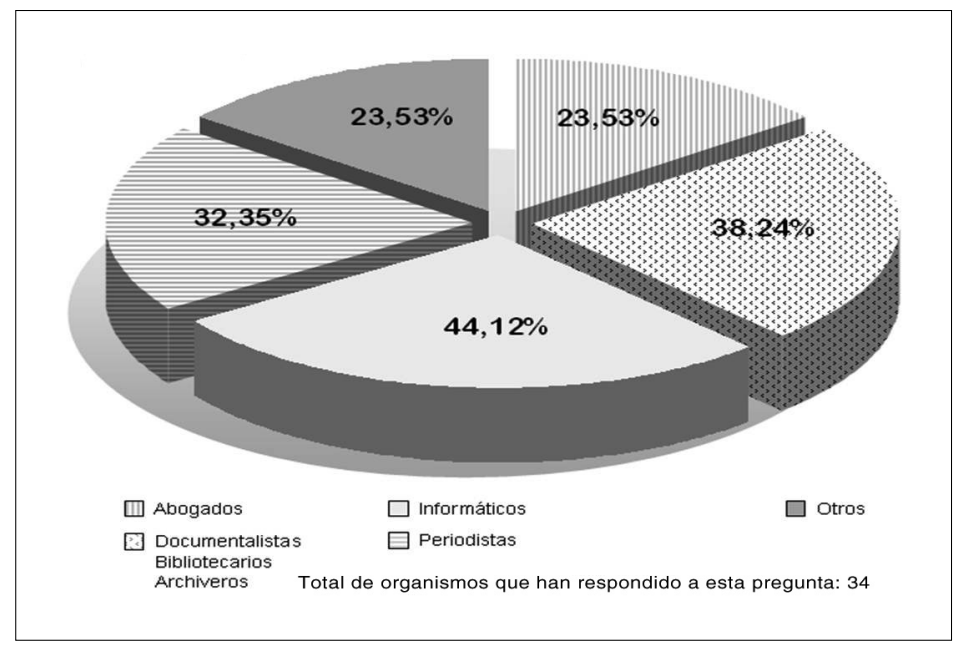


No se evidencia la existencia de un mercado de reutilización de la información del sector público, ya que es este mismo sector el principal demandante (figura 5) y, además, otro dato destacable es que más de la mitad de los organismos consultados no tienen establecidas tasas o precios públicos de los productos que ofrecen. Asimismo, la entrada en vigor de la Ley 37/2007, de 16 de noviembre, sobre reutilización de información del sector público, no ha supuesto un incremento del número de respuestas a las solicitudes de información de potenciales reutilizadores de la información del sector público.

\section{FIGURA 5}

\section{Demandantes de la información potencialmente reutilizable}

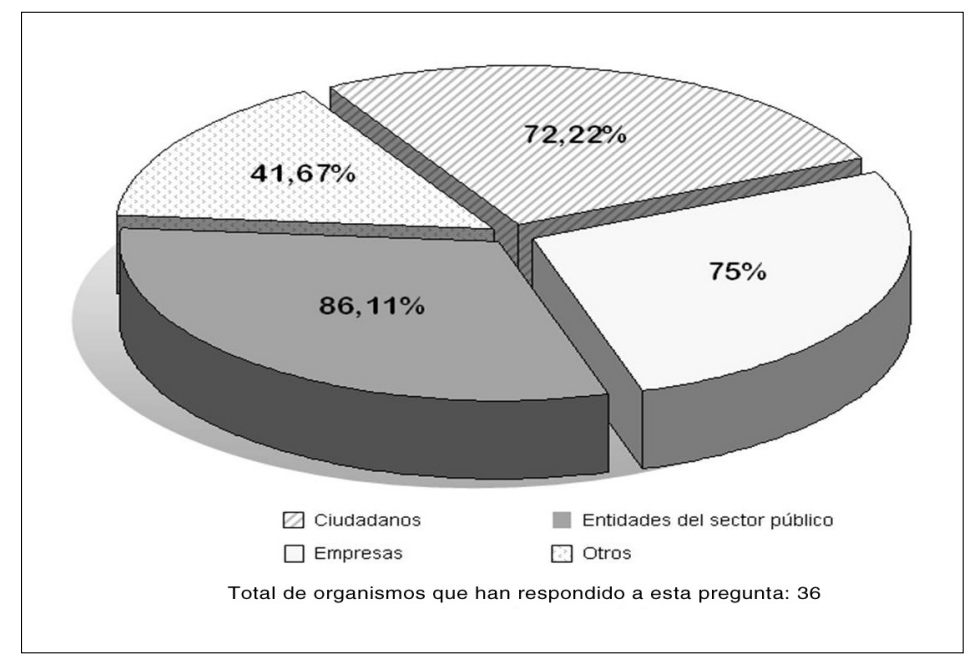

Los reutilizadores de la información generada por los organismos del sector público que han participado en el estudio se localizan fundamentalmente: en todas las Comunidades Autónomas del Estado español, en los países de la Unión Europea y en Iberoamérica. Dichos reutilizadores se pueden agrupar en los siguientes ámbitos: consultoras, editoriales jurídicas, medios de comunicación, organismos del sector público del ámbito español y europeo y Universidades.

\subsection{Acuerdos de explotación y condiciones de uso de la información reutilizable}

A tenor de las respuestas proporcionadas por los organismos del sector público, no existen acuerdos exclusivos. En este sentido, es rotunda la respuesta negativa $(91,89 \%)$ y el desconocimiento de su existencia. Sin embargo, contestan afirmativamente un $20 \%$ de los encuestados sobre disponibilidad de información pública 
acerca de términos de licencias (10\%) e información general de los acuerdos (10\%); el resto de organismos no tienen disponible esta información o lo desconocen.

Del análisis de los informes realizados en otros países europeos (es el caso de Alemania, Austria, Bélgica, Dinamarca, Polonia y República Checa) se deriva como conclusión la inexistencia de acuerdos exclusivos. En cambio, en el estudio de Francia se han detectado dos posibles acuerdos exclusivos y en el caso de Italia se han identificado un total de 7 potenciales acuerdos exclusivos.

Sobre las condiciones de uso de la información reutilizable o los acuerdos para la reutilización, con el mismo número de respuestas se sitúan quienes las ofrecen a través de las páginas Web y quienes alegan que la ofrecen previa solicitud (tabla VI). En la categoría de respuesta abierta para indicar otras vías de difusión se incluyen los Centros de Documentación.

\section{TABLA VI}

Vías a través de las que está disponible la información que se ofrece sobre acuerdos o condiciones de uso de la información potencialmente reutilizable

\begin{tabular}{l|c|c}
\hline & \% & Respuestas \\
\hline La información está disponible en la Web del organismo. & 66,67 & 16 \\
\hline Se ofrece la información previa solicitud. & 66,67 & 16 \\
\hline Está disponible a través de otras vías, por favor, precise cuáles. & 33,33 & 8 \\
\hline
\end{tabular}

Organismos que han respondido a esta pregunta: 24 .

Ningún órgano del Sector Público que ha participado en el estudio está dispuesto a facilitar documento alguno que contenga acuerdos exclusivos.

En la pregunta abierta a otros comentarios sobre reutilización de la información del Sector Público, de las respuestas ofrecidas emanan tres aspectos interesantes:

- No hay política de coordinación de la información para su reutilización, ni tampoco conciencia de que haya de haberla.

- Varias instituciones trabajan en estos momentos en el desarrollo de la legislación.

- Tienen condiciones claras para la reutilización de la información.

\section{Conclusiones y recomendaciones}

Del estudio realizado en el contexto español se deriva una conclusión incuestionable: el desconocimiento en profundidad de la Ley 37/2007 de 16 de noviembre, sobre reutilización de información del sector público y de todas las poten- 
cialidades que se pueden derivar de la misma. De dicha conclusión emana una recomendación que se sintetizaría en la necesidad de definir una política activa de difusión y comunicación del contenido y de las implicaciones de la Ley. A tenor del análisis de las conclusiones derivadas del estudio de la situación descrita en los informes de otros países europeos, podemos encontrar un claro paralelismo. Así, en el caso de Polonia se hace referencia al conocimiento limitado del contenido de la Directiva que tienen los propios representantes del sector público que han participado en el estudio. Igualmente, en el caso de Italia se pone de manifiesto que el conocimiento de la regulación europea sobre reutilización de la información pública es muy limitado. El informe alemán señala que la reacción general que se deriva de la encuesta realizada revela un alto grado de falta de conciencia sobre las políticas de reutilización de la información del sector público.

Al desconocimiento de la normativa sobre reutilización de la información se une la ausencia de una política de difusión de la reutilización de la información que debería conducir a una definición de iniciativas y acciones encaminadas a establecer mecanismos que garanticen la puesta en marcha de una estrategia de concienciación de la importancia de una difusión de la información con fines de reutilización. La relevancia de la puesta en marcha de dicha estrategia de concienciación adquiere mucho más peso si se tiene en cuenta que, tras la realización de la investigación, no se ha evidenciado la existencia de un mercado de reutilización de la información del sector público en nuestro país ni se ha detectado la conciencia entre los productores de las posibilidades y de las oportunidades que se podrían derivar de la potenciación de este mercado de la información del sector público. En virtud de esta situación, se imponen la necesidad de potenciar y definir medidas concretas para la creación y el desarrollo de este mercado como fuente de financiación y sostenimiento del sector público y como uno de los motores de la economía española. En comparación con la situación española descrita, del análisis de los informes de otros países europeos, sí podemos inferir la existencia de un mercado de reutilización de la información del sector público. En este sentido, en el informe italiano se incorpora una conclusión sobre la propia estructura del mercado de la información pública, de carácter diverso según el sector que se analice. Así, por ejemplo, se pone de manifiesto que el número de competidores en ciertos ámbitos (ejemplo, la información meteorológica) es menor que en otros sectores (es el caso de la información económica y geográfica). En el caso francés, se subraya la existencia de barreras para la libre competencia del mercado de la información pública del sector público, especialmente en los dominios de la información geográfica y económica y en el informe alemán se señala el ámbito de la información geográfica como líder en el mercado de la información pública en Alemania.

Hemos de señalar que una de las grandes dificultades en la realización del estudio se ha relacionado la siguiente situación: en ningún organismo del sector público participante en el estudio se ha encontrado un responsable y/o interlocutor para las cuestiones relacionadas con la reutilización de la información. En esta línea, consideramos que sería muy recomendable la creación, en cada orga- 
nismo del sector público, de una figura que asumiera las funciones propias de responsable y/o interlocutor en materia de reutilización (por ejemplo, en el informe de la República Checa se destaca que la mayoría de los organismos del sector público respondieron a la encuesta a través de sus departamentos legales). En este sentido, consideramos que, más allá de su relevancia en la participación específica en esta encuesta, la existencia de este interlocutor en materia de reutilización contribuiría a potenciar y desarrollar este mercado con vistas a facilitar la labor de los potenciales reutilizadores de la información del sector público.

Finalmente, hemos de destacan que de la realización de todos los informes se deriva la conclusión común de su contribución a la difusión de las políticas de reutilización de la información del sector público y a la necesidad de crear conciencia para fomentar el desarrollo de este sector con el fin último de la creación de empleo en este ámbito.

\section{Fuentes y bibliografía}

\section{Legislación citada}

Directiva 2003/98/CE del parlamento europeo y del consejo de 17 de noviembre de 2003 relativa a la reutilización de la información del sector público. Diario Oficial de la Unión Europea L 345 de 31/12/2003, 0090-0096. http://tinyurl.com/26spd2e [consultado el 1 de diciembre de 2010].

Ley $37 / 2007$, de 16 de noviembre, sobre reutilización de la información del sector público. BOE n. ${ }^{\circ} 276$ de 17/11/2007, 47160-47165. http://tinyurl.com/yax6dyr [consultado el 1 de diciembre de 2010].

Comisión de las Comunidades Europeas (2009): Comunicación de la comisión al parlamento europeo, al consejo, al comité económico y social europeo y al comité de las regiones: reutilización de la información del sector público - Revisión de la Directiva 2003/98/CE - Bruselas, 7.5.2009 COM(2009) 212 final. http://tinyurl.com/25ca8xe [consultado el 1 de diciembre de 2010].

\section{Estudios sobre Acuerdos exclusivos elaborados para la Comisión Europea}

A9 Consulting (2010): PSI: Identification of Potential Exclusive Agreements in Denmark.

Cities on Internet Association (2010): PSI: Identification of potential Exclusive Agreements, Poland.

DLA Piper UK LLP (2010): Country report assessment on exclusive PSI re-use arrangements in Belgium.

EPMA - Agentura pro evropské projekty a management (2010): PSI: Identification of potential Exclusive Agreement, Czech Republic.

GEOkomm IPEAG (2010): Public Sector Information (PSI): Identification of potential Exclusive Agreements - Germany.

GRUPO PUBLIDOC-UCM (2010): PSI: Identification of Potential Exclusive Agreements, Spain/Final Report. 
Preslmayr Rechtsanwälte O. G., y Dr. Rainer Knyrim (2010): Study PSI: Identification of potential Exclusive Agreements, Austria.

T33 Snc (2010): Study PSI: Identification of potential Exclusive Agreements, Italy.

Virginie Boillet, V., y Guerre, L. (2010): Psi re-use: Identification of potential exclusive agreements France Report.

Todos ellos pueden localizarse en: http://tinyurl.com/39f9l35 [consultado el 1 de diciembre de 2010].

\section{Bibliografía especializada}

Ramos Simón, L. F.; Mendo Carmona, C., y Arquero Avilés, R. (2009): La producción informativa y documental del Estado: hacia un inventario de los recursos públicos. Revista Española de Documentación Científica, enero-marzo, vol. 32 (1), 40-59.

\section{ANEXOS}

\subsection{Relación de organismos del sector público a los que se envió el cuestionario}

\section{Información económica}

\begin{tabular}{l}
\hline Agencia de Desarrollo Económico de la Rioja \\
\hline Agencia de Innovación y Desarrollo de Andalucía \\
\hline Agencia del Conocimiento y de la Tecnología \\
\hline Agencia Gallega de Desarrollo Rural \\
\hline Banco de España \\
\hline Centro Español de Metrología \\
\hline Comisión Nacional del Mercado de Valores \\
\hline Consejería de Medio Ambiente, Vivienda y Ordenación del Territorio de la Comunidad de Madrid \\
\hline Consejo de Cámaras de Comercio e Industria de Castilla y León (COCICYL) \\
\hline Consejo Superior de Cámara de Comercio \\
\hline Ente Vasco de la Energía (EVE) \\
\hline Fundación Española para la Innovación de la Artesanía \\
\hline Fundación INCYDE \\
\hline Generalitat Valenciana \\
\hline Instituto Catalán de Crédito Agrario \\
\hline
\end{tabular}




\section{Información económica}

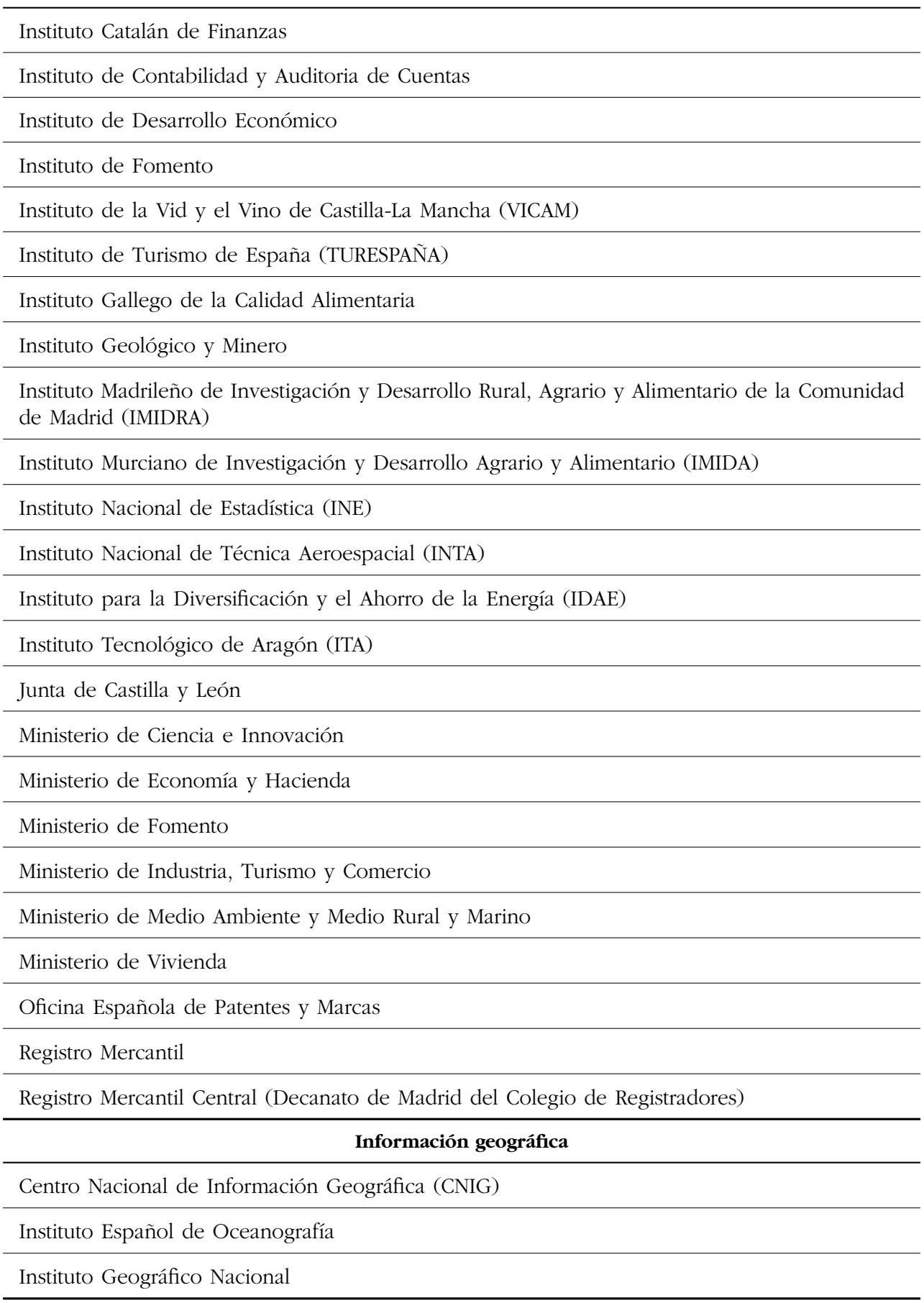




\section{Información legal}

\begin{tabular}{l} 
Agencia de Protección de Datos \\
\hline BOE \\
\hline Comunidad de Madrid \\
\hline Congreso de los Diputados \\
\hline Consejo de Estado \\
\hline Consejo General del Poder Judicial \\
\hline Junta de Castilla y León \\
\hline Comunidad Autónoma de La Rioja \\
\hline Ministerio de Justicia \\
\hline Ministerio de la Presidencia
\end{tabular}

\section{Información social}

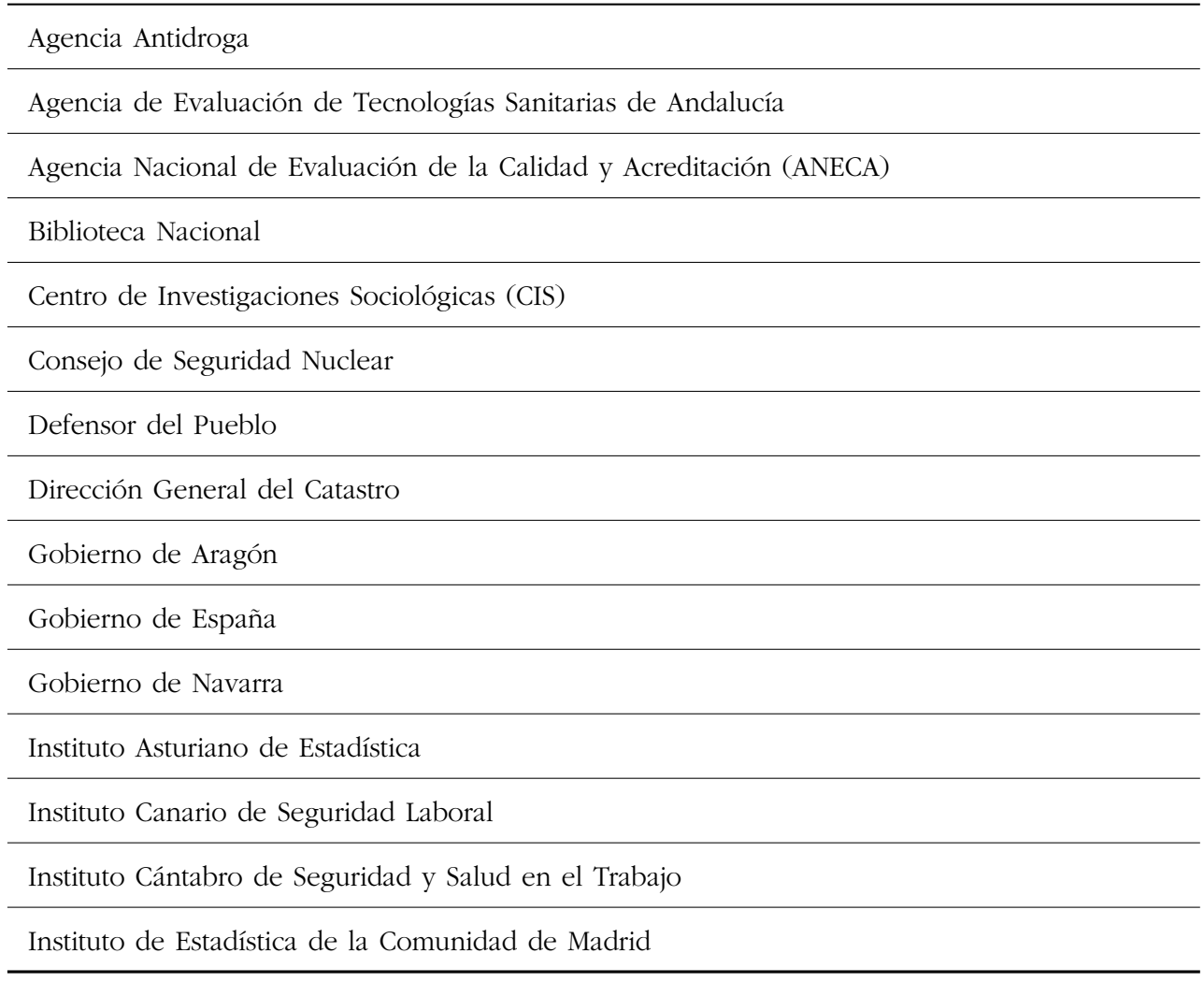




\section{Información social}

Instituto de Estadística de las Islas Baleares

Instituto de Estudios Ceutíes

Instituto de Estudios Riojanos

Instituto de la Juventud

Instituto de la Mujer

Instituto de Mayores y Servicios Sociales (IMSERSO)

Instituto Madrileño de Administraciones Públicas (IMAP)

Instituto Nacional de Administración Pública

Instituto Navarro para la Igualdad

Instituto Vasco de Administración Pública (IVAP)

Junta de Extremadura

Ministerio de Asuntos Exteriores y Cooperación

Ministerio de Cultura

Ministerio de Educación

Ministerio de Igualdad

Ministerio de Política Territorial

Ministerio de Sanidad y Política Social

Ministerio del Interior

Museo Nacional del Prado

Región de Murcia

Región de Murcia: Servicio de Publicaciones y Estadística

Senado

Xunta de Galicia

Información sobre transportes

ADIF

AENA

Centro de Estudios y Experimentación de Obras Públicas (CEDEX)

Consorci de l'Autoritat del Transport Metropolità (ATM) 
Información sobre transportes

Departamento de Interior del Gobierno Vasco

Dirección General de Tráfico

Ferrocarriles de la Generalitat de Catalunya (FGC)

Información meteorológica

Agencia Estatal de Meteorología (AEMET)

Euskalmet / Dirección de Meteorología y Climatología Gobierno Vasco

Servei Meteorològic de Catalunya 